\title{
BULGE AND DISK: A SIMPLE SELF-GRAVITATING MODEL
}

\section{S.A. KUTUZOV and L.P. OSSIPKOV}

\author{
St. Petersburg University, Russia
}

A method of finding the distribution function of some steady-state axially symmetrical mass models was suggested by Ossipkov, Kutuzov (1987). The models consist of a disk embedded in a bulge (halo). The total potential $\phi(R, z)=\phi_{0} \varphi(\xi)$, with $\phi_{0}=\phi(0,0), \varphi(\xi)$ arbitrary,

$$
\xi^{2}=\rho^{2}+2 \mu|\zeta|+\zeta^{2}, \quad \mu=\text { const } \geq 0
$$

and $(R ; z)$ resp. $(\rho, \zeta)$ dimensional resp. dimensionless cylindrical coordinates respectively. The total dimensionless configuration density has the following form:

$$
\nu(\rho, \zeta)=\nu_{b}(\xi)+\delta(\zeta) \sigma_{d}(\rho)
$$

where $\nu_{b}$ is the bulge density, $\sigma_{d}$ is the disk surface density and $\delta(\zeta)$ is the Dirac delta function. The bulge lenslike equidensity surfaces coincide with equipotential ones. The parameter $\mu$ determines their flattening: they are spherical if $\mu=0$ and flat if $\mu=\infty$. The following expressions are found for bulge and disk densities:

$$
\nu_{b}(\xi)=3 \omega^{2}(\xi)+2\left(\mu^{2}+\xi^{2}\right) \frac{d \omega^{2}}{d\left(\xi^{2}\right)}, \quad \sigma_{d}(\rho)=2 \mu \omega^{2}(\rho)
$$

where $\omega^{2}(\xi)=-2 d \varphi(\xi) / d\left(\xi^{2}\right), \omega(\rho)$ is a dimensionless circular frequency. Both densities are connected with each other by means of the equations (3).

The distribution function has the following form:

$$
\Psi(E, e, h)=\Psi_{b}(E)+\delta(\zeta) \delta\left(\mathcal{U}_{\zeta}\right) \Psi_{d}(e, h)
$$

where $E=\varphi(\xi)-\left(\mathcal{U}_{\rho}^{2}+\mathcal{U}_{\theta}^{2}+\mathcal{U}_{\zeta}^{2}\right) / 2$ and $e=\varphi(\rho)-\left(\mathcal{U}_{\rho}^{2}+\mathcal{U}_{\theta}^{2}\right) / 2$ are the energy integrals of spatial and flat motion respectively, $h=\rho \mathcal{U}_{\theta}$ is an integral of angular momentum, $\mathcal{U}_{\rho}, \mathcal{U}_{\theta}, \mathcal{U}_{\zeta}$ are dimensionless velocity components in cylindrical coordinates. The bulge does not rotate and its velocity distribution is isotropic. The bulge distribution function can be found as a solution of the integral equation:

$$
\sqrt{8} \pi^{2} \Psi_{b}(E)=\frac{d^{2}}{d E^{2}}\left(\int_{0}^{E}(E-\varphi)^{\frac{1}{2}} G(\varphi) d \varphi\right)
$$

Here $G(\varphi)=\nu_{b}(\xi(\varphi))$ is an augmented density (Dejonghe 1987) but the inverted potential law $\xi(\varphi)$ is supposed to be a single-valued function.

The disk phase density is decomposed into even and odd components with respect to the azimuthal velocity (and $h$ ):

$$
\Psi_{d}(e, h)=\Psi_{+}(e, h)+\Psi_{-}(e, h)
$$

The even component can be found on the basis of the surface density by known methods (Kalnajs 1976, Ossipkov 1978). In particular if it depends on the $e$ only then (Dekker 1976) 


$$
2 \pi \Psi_{+}(e)=\frac{d g(e)}{d e}
$$

where $g(\varphi)=\sigma_{d}(\rho(\varphi))$ is an augmented surface density. The odd component is determined by a rotation law $\left\langle\mathcal{U}_{\theta}\right\rangle=\rho \Omega(\rho)$. It is assumed to be separable in $e$ and $h$, that gives rise to

$$
\Psi_{-}(e, h)=u(e) h, \quad 2 \pi u(e)=\frac{d^{2} f(e)}{d e^{2}}
$$

where $f(\varphi)=g(\varphi) \Omega(\rho(\varphi))$.

As an example we consider the particular cases of the Kuzmin-Malasidze (1969) potential law

$$
\varphi(\xi)=\alpha\left(\alpha-1+\left(1+\kappa \xi^{2}\right)^{\frac{1}{2}}\right)^{-1}, \quad \alpha, \kappa=\text { const }>0
$$

and suggest the rotation law

$$
\Omega(\rho(\varphi))=a\left[1-b \varphi^{p}(1-\varphi)^{q}\right] \varphi^{\tau}, \quad a, b, p ; q, \tau>0
$$

which allows a rotation curve with one minimum and flat outer part. Some restrictions on the functions and the parameters are established from the condition of non-negativity of the $\Psi_{d}(e, h)$. The rotation velocity $\left\langle\mathcal{U}_{\theta}\right\rangle$ has to be considerably smaller everywhere than the circular one $\mathcal{U}=\rho \omega(\rho)$. In the case of $\alpha=\kappa \mu^{2}=1$ there is a pure Kuzmin-Toomre disk (Kuzmin 195.3, Toomre 1963) without bulge. Then $\tau>3 / 2$ and $\alpha \leq 0.30$ for $\tau=1.6, b=0$.

\section{References}

Dejonghe, H., 1987, Inst.Adv.Studies Preprint, IASSNS-AST 87/10

Dekker, E., 1976, Phys.Rep., 24c, 315

Kalnajs, A., 1976, Ap.J., 205, 751

Kuzmin, G.G., 1953, Isv.Akad.Nauk Est.SSR, 5, 369 (Tartu Teated, No 1)

Kuzmin, G.G., Malasidze, G.A.:1969 (1970), Tartu Publ., 38, 181

Ossipkov, L.P. 1978, Pis'ma Astr.Zh., 4, 70

Ossipkov, L.P., Kutuzov, S.A. 1987, Astrofizika, 27, 523

Toomre, A. 1963. Ap.J., 138, 385 